\title{
Some Properties of the Intersection Graph for Finite Commutative Principal Ideal Rings
}

\author{
Emad Abu Osba, ${ }^{1}$ Salah Al-Addasi, ${ }^{2}$ and Omar Abughneim ${ }^{1}$ \\ ${ }^{1}$ Department of Mathematics, The University of Jordan, Amman 11942, Jordan \\ ${ }^{2}$ Department of Mathematics, Hashemite University, Zarqa 13115, Jordan
}

Correspondence should be addressed to Emad Abu Osba; eabuosba@ju.edu.jo

Received 28 May 2014; Accepted 10 September 2014; Published 25 September 2014

Academic Editor: Johannes Hendrik Hattingh

Copyright (C) 2014 Emad Abu Osba et al. This is an open access article distributed under the Creative Commons Attribution License, which permits unrestricted use, distribution, and reproduction in any medium, provided the original work is properly cited.

Let $R$ be a commutative finite principal ideal ring with unity, and let $G(R)$ be the simple graph consisting of nontrivial proper ideals of $R$ as vertices such that two vertices $I$ and $J$ are adjacent if they have nonzero intersection. In this paper we continue the work done by Abu Osba. We calculate the radius, eccentricity, domination number, independence number, geodetic number, and the hull number for this graph. We also determine when $G(R)$ is chordal. Finally, we study some properties of the complement graph of $G(R)$.

\section{Introduction}

All rings are assumed to be finite commutative principal ideal rings with unity 1.

For each vertex $x$ in a graph $\Gamma$, let $\operatorname{deg}(x)$ be the number of vertices adjacent to $x$ and let $N(x)$ be the set of vertices adjacent to $x$ in $\Gamma$. For any undefined graph theoretical terms, the reader may consult [1].

Let $F=\left\{A_{j}: j \in J\right\}$ be a family of nonempty sets. The intersection graph $G(F)$ defined on $F$ is a simple graph whose vertex set is $F$ and two vertices $A_{j}$ and $A_{i}$ are adjacent if $A_{j} \neq$ $A_{i}$ and $A_{j} \cap A_{i} \neq \phi$. Many authors worked on the graphs $G(F)$ when the members of $F$ have an algebraic structure; see, for example, [2-7].

The intersection graph $G(R)$ of ideals of a ring $R$ is a simple graph whose vertices are the nontrivial proper ideals and two vertices $I, J$ are adjacent if $I \neq J$ and $I \cap J \neq\{0\}$. Note that if $R$ is a field, then $G(R)$ is the null graph which has no vertices. Extending statements to the null graph would introduce unnecessary distractions, so we ignore the null graph; that is, all rings are assumed to be nonfields except when stated explicitly.

In this paper we consider the intersection graph $G(R)$ of nontrivial proper ideals of a finite commutative principal ideal ring $R$ with unity 1 . If $R$ is a finite commutative ring, then it can be written as a product of local rings; see [8]. If $R=\prod R_{j}$ and $R$ has a unity, then any ideal $I$ of $R$ can be written as $\prod I_{j}$, where $I_{j}$ is an ideal in $R_{j}$ for each $j$, while if $R$ has no unity, then this needs not to be true; see [9, page 135]. If $R$ is a finite local principal ideal ring with maximal ideal $M=a R$, then the ideals of $R$ are $M=a R, M^{2}=a^{2} R$, and $M^{3}=a^{3} R, \ldots, M^{n-1}=a^{n-1} R, M^{n}=\{0\}$ for some $n \in \mathbb{N}$; see the proof of Proposition 8.8 in [10].

This study is a continuation of the study in [2], where the author used the fact that if $R$ is a local ring with maximal ideal $M$, then there exists $n \in \mathbb{N}$ such that $M^{n}=\{0\}$ but $M^{n-1} \neq\{0\}$, to define $\operatorname{Nilpotency}(R)=n$. If $R$ is a nonlocal ring such that $R=\prod_{j=1}^{n} R_{j}$, where $R_{j}$ is a local ring with $\operatorname{Nilpotency}\left(R_{j}\right)=n_{j}$ for $j=1,2, \ldots, n$, then $R$ has $\left(\prod_{j=1}^{n}\left(n_{j}+1\right)\right)-2$ nontrivial proper ideals. The author characterized when $G(R)$ is Eulerian, Hamiltonian, planar, or bipartite.

In this paper we will continue the investigation of properties of the intersection graph and calculate the radius, eccentricity, dominating number, independence number, geodetic number, and the hull number; we also determine when $G(R)$ is chordal. We conclude this paper by a study of some properties of the complement graph of $G(R)$. 


\section{The $n$-Cube}

The $n$-cube $Q_{n}(n \geq 1)$ is the graph whose vertex set is the set of all binary $n$-tuples, where two $n$-tuples are adjacent if and only if they differ in precisely one coordinate.

Theorem 1. Let $R=\prod_{j=1}^{n} R_{j}$, where $R_{j}$ is a finite local principal ideal ring for each $j$. Then $G(R)$ contains a subgraph isomorphic to $Q_{n}-\{(0,0, \ldots, 0),(1,1, \ldots, 1)\}$. In particular, if each $R_{j}$ is a field, then this subgraph is a spanning subgraph of $G(R)$.

Proof. Let $A=\left\{\prod_{j=1}^{n} I_{j} \in G(R): I_{j} \in\left\{\{0\}, R_{j}\right\}\right\}$. Consider the one-to-one correspondence $f: Q_{n}-\{(0,0, \ldots, 0),(1,1$, $\ldots, 1)\} \rightarrow A$, defined by $f\left(\left(b_{1}, b_{2}, \ldots, b_{n}\right)\right)=\prod_{j=1}^{n} I_{j}$, where

$$
I_{j}= \begin{cases}R_{j} & \text { if } b_{j}=1 \\ \{0\} & \text { if } b_{j}=0\end{cases}
$$

If $\left(b_{1}, b_{2}, \ldots, b_{n}\right)$ and $\left(c_{1}, c_{2}, \ldots, c_{n}\right)$ are two adjacent vertices of $Q_{n}-\{(0,0, \ldots, 0),(1,1, \ldots, 1)\}$, then there exists a unique $j_{1} \in\{1,2, \ldots, n\}$ such that $\left\{b_{j_{1}}, c_{j_{1}}\right\}=\{0,1\}$; say $b_{j_{1}}=1$ and $c_{j_{1}}=0$. Since $\left(c_{1}, c_{2}, \ldots, c_{n}\right) \neq(0,0, \ldots, 0)$, we can find $j_{2} \in$ $\{1,2, \ldots, n\}-\left\{j_{1}\right\}$ such that $c_{j_{2}}=1$. But $b_{j_{2}}=c_{j_{2}}$, and hence $f\left(\left(b_{1}, b_{2}, \ldots, b_{n}\right)\right)=\prod_{j=1}^{n} I_{j}$ and $f\left(\left(c_{1}, c_{2}, \ldots, c_{n}\right)\right)=\prod_{j=1}^{n} T_{j}$ are adjacent in $G(R)$ because $I_{j_{2}}=T_{j_{2}}=R_{j_{2}}$. Therefore the subgraph of $G(R)$ induced by $A$ contains a copy of $Q_{n}-$ $\{(0,0, \ldots, 0),(1,1, \ldots, 1)\}$. Note that the set $A$ consists of all vertices of $G(R)$ when $R$ is a product of fields.

The previous result shows that $G(R)$ contains $Q_{n}-$ $\{(0,0, \ldots, 0),(1,1, \ldots, 1)\}$ as a subgraph. This subgraph is induced only when $n<3$. If $R=\prod_{j=1}^{n} R_{j}$, where $n \geq 3$ and $R_{j}$ is a local ring for each $j$, then $I=\prod_{j=1}^{n} I_{j}$ and $T=\prod_{j=1}^{n} T_{j}$, where $I_{1}=R_{1}, I_{2}=R_{2}$, and $I_{j}=\{0\}$ for $j \neq 1,2$ and $T_{1}=R_{1}, T_{3}=R_{3}$, and $T_{j}=\{0\}$ for $j \neq 1,3$ are two elements of $A$. Clearly, $I$ and $T$ are adjacent in the subgraph of $G(R)$ induced by $A$, while $f^{-1}(I)$ and $f^{-1}(T)$ are not adjacent in $Q_{n}-\{(0,0, \ldots, 0),(1,1, \ldots, 1)\}$.

\section{Dominating Sets and Numbers of $G(R)$}

In a graph $\Gamma$, a dominating set is a set of vertices $A$ such that every vertex outside $A$ is adjacent to at least one vertex in $A$. The domination number of a graph $\Gamma$, denoted by $\gamma(\Gamma)$, is the smallest number of the form $|A|$, where $A$ is a dominating set.

If $R$ is a finite local principal ideal ring with maximal ideal $M$, then $G(R)$ is complete, and so $A=\{M\}$ is a dominating set, and $\gamma(G(R))=1$. Assume that $R$ is nonlocal and $R=$ $\prod_{j=1}^{n} R_{j}$, where $R_{j}$ is local for each $j$. We have two cases.

Case 1. One factor, say $R_{1}$, is not a field, with maximal ideal $M$. Let $I=M \times R_{2} \times \cdots \times R_{n}$. Then $A=\{I\}$ is a dominating set, and $\gamma(G(R))=1$.

Case 2. $R$ is a product of fields. Let $I$ be a nontrivial proper ideal in $R$. Then $I$ is of the form $\prod_{j=1}^{n} I_{j}$, where $I_{k}=\{0\}$ for at least one $k$. Let $J=\prod_{j=1}^{n} J_{j}$ such that $J_{k}=R_{k}$ and $J_{j}=\{0\}$ for $j \neq k$. Then $I \cap J=\{0\}$, and so, $\{I\}$ cannot be a dominating set. Thus $\gamma(G(R))>1$. Now, let $I=\{0\} \times R_{2} \times \cdots \times R_{n}$ and $J=R_{1} \times\{0\} \times \cdots \times\{0\}$. Then $A=\{I, J\}$ is a dominating set, and $\gamma(G(R))=2$. Thus we have the following result.

Theorem 2. For any finite principal ideal ring $R$ which is not a field, $\gamma(G(R))=1$ except when $R$ is a product of fields; one has $\gamma(G(R))=2$.

\section{Radius and Center of $G(R)$}

For a graph $\Gamma$, the eccentricity of a vertex $f$ in $\Gamma$ is $e(f)=$ $\operatorname{Max}\{d(f, g): g$ is a vertex in $\Gamma\}$. A center of $\Gamma$ is a vertex $f_{0}$ with smallest eccentricity. The eccentricity $e\left(f_{0}\right)$ is called the radius of $\Gamma$ and is denoted by $\rho(\Gamma)$.

Now, we calculate the radius of $G(R)$.

Theorem 3. For any finite principal ideal ring $R$ which is not a field, one has

$$
\rho(G(R))= \begin{cases}0 & \text { if } R \text { is local with Nilpotency }(R)=2 \\ \infty & \text { if } R \text { is a product of two fields } \\ 2 & \text { if } R \text { is a product of more than two fields } \\ 1 & \text { otherwise. }\end{cases}
$$

Proof. Let $R$ be a local ring with $\operatorname{Nilpotency}(R)=2$. Then $G(R)$ is $K_{1}$ and so $\rho(G(R))=0$.

Assume now that $R$ is local with $\operatorname{Nilpotency}(R)>2$ or $R$ is not a product of fields. If $R$ is a local ring or $R$ is not a product of fields, then there exists an ideal $I$ in $G(R)$ such that $I$ is adjacent to every other ideal in $G(R)$ and so, $e(I)=$ $1=\rho(G(R))$. If $R=F_{1} \times F_{2}$ is a product of two fields, then $I=F_{1} \times\{0\}$ and $J=\{0\} \times F_{2}$ are the only vertices in $G(R)$ and they are nonadjacent. So $\rho(G(R))=e(J)=e(I)=\infty$. Let $R=\prod_{j=1}^{n} F_{j}$ be a product of fields with $n>2$, and $I, J \in G(R)$ such that $I \cap J=\{0\}$. If $I+J \neq R$, then $I I+J_{-} J$ is a path in $G(R)$. If $I+J=R$, then $I=\prod_{j=1}^{n} I_{j}$, where $I_{j}=\{0\}$ or $F_{j}$ and $J=\prod_{j=1}^{n} J_{j}$, where $J_{j}=\{0\}$ or $F_{j}$. Let $k$ be the least element in $\{1,2, \ldots, n\}$ such that $I_{k}=F_{k}$ and let $s$ be the least element in $\{1,2, \ldots, n\}$ such that $J_{s}=F_{s}$. It is clear that $k \neq s$. Define $L=\prod_{j=1}^{n} L_{j}$ such that

$$
L_{j}= \begin{cases}F_{k} & j=k \\ F_{s} & j=s \\ \{0\} & \text { otherwise. }\end{cases}
$$

Then $L$ is a vertex in $G(R)$ and $I_{-} L_{-} J$ is a path in $G(R)$. Hence $d(I, J)=2$ and $e(I)=2$. Since there is no ideal in $G(R)$ that is adjacent to every other ideal, we have $\rho(G(R))=2$.

Note that if $R$ is a local ring with maximal ideal $M$, then $M$ is a center for $G(R)$, and if $R=\prod_{j=1}^{n} R_{j}$, where $R_{1}$ is a local ring with maximal ideal $M \neq\{0\}$, then $M \times \prod_{j=2}^{n} R_{j}$ is a center for $G(R)$, while if $R=\prod_{j=1}^{n} F_{j}$, where $F_{j}$ is a field for each $J$ and $n>2$, then $\{0\} \times \prod_{j=2}^{n} F_{j}$ is a center for $G(R)$. 
The following result was proved in [2] and will be used in the proof of the next theorem.

Lemma 4. For any finite principal ideal ring $R$ which is not a field, one has

$$
\operatorname{diam}(G(R))= \begin{cases}0 & \text { if } R \text { is local with Nilpotency }(R)=2 \\ 1 & \text { if } R \text { is local with Nilpotency }(R)>2 \\ \infty & \text { if } R \text { is a product of two fields } \\ 2 & \text { otherwise. }\end{cases}
$$

The subgraph of a graph $\Gamma$ induced by the set of centers of $\Gamma$ is denoted by $\operatorname{Cen}(\Gamma)$. The graph $\Gamma$ is called self-centered if $\operatorname{Cen}(\Gamma)=\Gamma$.

Theorem 5. Let $R$ be a finite principal ideal ring. The graph $G(R)$ is self-centered if and only if $R$ is local or a product of fields.

Moreover, if $R=\prod_{j=1}^{n} R_{j}$, where $n \geq 2, R_{j}$ is a finite principal ideal local ring for each $j$, and there is at least one $R_{j}$ having nilpotency greater than 1 , then the vertex set of $\operatorname{Cen}(G(R))$ is $\left\{\prod_{j=1}^{n} I_{j}: I_{j} \neq\{0\}\right.$ for each $\left.j\right\}$ and $\operatorname{Cen}(G(R))=$ $K_{\left(\prod_{j=1}^{n} n_{j}\right)-1}$, where $n_{j}=\operatorname{Nilpotency}\left(R_{j}\right)$.

Proof. The graph $G(R)$ is self-centered when its diameter and radius are equal. Thus, by Lemma 4 and Theorem 3, $G(R)$ is self-centered when $R$ is local or $R$ is a product of $n$ fields with $n \geq 2$. So, suppose that $R=\prod_{j=1}^{n} R_{j}$, where $n \geq 2, R_{j}$ is a local ring for each $j$, and there is at least one $R_{j}$ having nilpotency greater than 1 . Then, by Theorem 3, $G(R)$ has radius 1 . Any vertex $I=\prod_{j=1}^{n} I_{j}$ with $I_{j} \neq\{0\}$ for each $j$ is adjacent to every other vertex of $G(R)$. Thus $I$ is a center of $G(R)$. Now let $J=$ $\prod_{j=1}^{n} J_{j}$ with $J_{j_{0}}=\{0\}$ for some $j_{0} \in\{1,2, \ldots, n\}$ be a vertex of $G(R)$. Take the vertex $L=\prod_{j=1}^{n} L_{j}$ with $L_{j_{0}}=R_{j_{0}}$ and $L_{j}=\{0\}$ for every $j \neq j_{0}$. Obviously, $J$ and $L$ are not adjacent and hence $J$ is not a center of $G(R)$. Thus the vertex set of $\operatorname{Cen}(G(R))$ is $\left\{\prod_{j=1}^{n} I_{j}: I_{j} \neq\{0\}\right.$ for each $\left.j\right\}$ which has cardinality $\left(\prod_{j=1}^{n} n_{j}\right)-$ 1 , where $n_{j}=\operatorname{Nilpotency}\left(R_{j}\right)$. The fact that $\operatorname{Cen}(G(R))$ is a complete graph follows directly from the fact that $G(R)$ has radius 1 .

\section{Independence Number of $G(R)$}

An independent vertex set of a graph $\Gamma$ is a set of vertices such that no two of them are adjacent in $\Gamma$. The vertex independence number $\alpha(\Gamma)$ of a graph, often called simply the independence number, is the cardinality of the largest (vertex) independent set.

Theorem 6. If $R=\prod_{j=1}^{n} R_{j}$, where $R_{j}$ is a finite local principal ideal ring for each $j$, then $\alpha(G(R))=n$.

Proof. If $R$ is local, then $\alpha(G(R))=1=n$. So assume that $n>1$. Suppose that $M$ is an independent set of $G(R)$. Suppose that $N=\prod_{j=1}^{n} N_{j} \in M$, where $N_{j_{1}} \neq\{0\}, N_{j_{2}} \neq\{0\}$, and $1 \leq$ $j_{1} \neq j_{2} \leq n$. Define $X=\prod_{j=1}^{n} X_{j}$, where $X_{j_{1}}=N_{j_{1}}, X_{j}=\{0\}$ for $j \neq j_{1}$ and $Y=\prod_{j=1}^{n} Y_{j}$, where $Y_{j_{2}}=N_{j_{2}}, Y_{j}=\{0\}$ for $j \neq j_{2}$. Observe that $(M-\{N\}) \cup\{X, Y\}$ is an independent set whose cardinality is greater than the cardinality of $M$. So, an independent set with maximum cardinality cannot contain any element $N=\prod_{j=1}^{n} N_{j}$, where $N_{j} \neq\{0\}$ for two or more indices. Thus $\mathscr{I}=\left\{\prod_{j=1}^{n} I_{j}: I_{k}=R_{k}\right.$ for exactly one $k$ and $I_{j}=\{0\}$ for $\left.j \neq k\right\}$ is an independent set with maximum cardinality in $G(R)$ and $\alpha(G(R))=n$.

\section{Geodetic and Hull Numbers of $G(R)$}

Let $u, v$ be two vertices of a connected graph $\Gamma$. A shortest path between $u$ and $v$ is called a $u-v$ geodesic. The set of all vertices in $\Gamma$ that lie on a $u-v$ geodesic is denoted by $I[u, v]$. For any subset $S$ of $G$, let $I[S]=\bigcup_{u, v \in S} I[u, v]$. If $I[S]=V(\Gamma)$, then $S$ is called a geodetic set of $\Gamma$. The minimum cardinality of a geodetic set of $\Gamma$ is called the geodetic number of $\Gamma$ and this number is denoted by $g(\Gamma)$. A subset $S$ of $V(\Gamma)$ is convex if $I[S]=S$. If $A$ is a subset of $V(\Gamma)$, then the convex hull of $A$ (denoted by $[A]$ ) is the smallest convex set in $\Gamma$ containing $A$. If $[A]=V(\Gamma)$, then $A$ is called a hull set of $\Gamma$. The smallest cardinality of a hull set of $\Gamma$ is called the hull number of $\Gamma$ and is denoted by $h(\Gamma)$. It is clear that $h(\Gamma) \leq g(\Gamma)$ for any graph $\Gamma$. In this section, we find the geodetic and hull numbers of $G(R)$.

A vertex $v$ in a graph $\Gamma$ is called an extreme vertex if the subgraph induced by its neighbors is complete. The set of extreme vertices of $\Gamma$ is denoted by $\operatorname{Ext}(\Gamma)$. The following lemma can be found in [11] or [12].

Lemma 7. Every geodetic set (resp., hull set) of $\Gamma$ contains $\operatorname{Ext}(\Gamma)$.

Note that if $R$ is a local ring with $\operatorname{Nilpotency}(R)=n$, then $G(R)$ is complete, and so $g(G(R))=n-1=h(G(R))$. The following lemma determines the set of extreme vertices of $G(R)$, where $R$ is a nonlocal ring.

Lemma 8. Let $R$ be a product of finite local principal ideal rings, and $T=\left\{\prod_{j=1}^{n} I_{j}: I_{j} \neq\{0\}\right.$ for exactly one $\left.j\right\}$. Then $T$ is the set of extreme vertices of $G(R)$.

Proof. Let $L=\prod_{j=1}^{n} L_{j}$ and $K=\prod_{j=1}^{n} K_{j}$ be two vertices that are adjacent to $I=\prod_{j=1}^{n} I_{j}$, where $I_{j_{1}} \neq\{0\}$ for $j_{1}$ only. Then $L_{j_{1}} \neq\{0\}$ and $K_{j_{1}} \neq\{0\}$. Thus $L$ and $K$ are adjacent. Hence the subgraph induced by the neighbors of $I$ is a complete subgraph and $I$ is an extreme vertex. Let $N=\prod_{j=1}^{n} N_{j}$, where $N_{j_{2}} \neq\{0\}, N_{j_{3}} \neq\{0\}$, with $1 \leq j_{2} \neq j_{3} \leq n$. Define $X=\prod_{j=1}^{n} X_{j}$, where $X_{j_{2}}=N_{j_{2}}, X_{j}=\{0\}$ for $j \neq j_{2}$ and $Y=\prod_{j=1}^{n} Y_{j}$, where $Y_{j_{3}}=N_{j_{3}}, Y_{j}=\{0\}$ for $j \neq j_{3}$. Observe that $X$ and $Y$ are not adjacent, but both are adjacent to $N$. So, $N$ is not an extreme vertex of $G(R)$. Thus, $T$ is the set of extreme vertices of $G(R)$.

Theorem 9. Let $R$ be a product of finite local principal ideal rings. Then the set $T=\left\{\prod_{j=1}^{n} I_{j}: I_{j} \neq\{0\}\right.$ for exactly one $\left.j\right\}$ is a geodetic set of $G(R)$ and $g(G(R))=\sum_{j=1}^{n} n_{j}$, where $n_{j}=$ $\operatorname{Nilpotency}\left(R_{j}\right)$. 
Proof. Let $N=\prod_{j=1}^{n} N_{j}$ be any vertex of $G(R)-T$. Then there are $j_{1}$ and $j_{2}$, where $N_{j_{1}} \neq\{0\}, N_{j_{2}} \neq\{0\}$, with $1 \leq j_{1} \neq j_{2} \leq n$. Take $X, Y \in T$, where $X_{j_{1}}=N_{j_{1}}$ and $Y_{j_{2}}=N_{j_{2}}$. Then $X \_N_{-} Y$ is a geodesic that contains $N$. Use Lemmas 7 and 8 to get the result.

According to Theorem 9 and Lemmas 7 and 8, the hull number of $G(R)$ is equal to the geodetic number of $G(R)$. Also the set $T$ is a hull set of $G(R)$. We state that in the following corollary.

Corollary 10. Let $R$ be a product of finite local principal ideal rings. Then the set $T=\left\{\prod_{j=1}^{n} I_{j}: I_{j} \neq\{0\}\right.$ for exactly one $\left.j\right\}$ is a hull set of $G(R)$ and $h(G(R))=\sum_{j=1}^{n} n_{j}$, where $n_{j}=$ $\operatorname{Nilpotency}\left(R_{j}\right)$.

\section{When Is $G(R)$ Chordal?}

A graph $\Gamma$ is chordal if it has no induced cycle of length greater than 3.

Theorem 11. Let $R$ be a finite principal ideal ring which is not a field. The graph $G(R)$ is chordal if and only if $R$ is the product of at most three local rings.

Proof. If $R=\prod_{j=1}^{n} R_{j}$, where $n \geq 4$ and $R_{j}$ is a local ring for each $j$, then the four vertices $R_{1} \times R_{2} \times \prod_{j=3}^{n}\{0\}, R_{1} \times\{0\} \times$ $R_{3} \times \prod_{j=4}^{n}\{0\},\{0\} \times\{0\} \times R_{3} \times R_{4} \times \prod_{j=5}^{n}\{0\}$, and $\{0\} \times R_{2} \times\{0\} \times$ $R_{4} \times \prod_{j=5}^{n}\{0\}$ induce a 4 -cycle $G(R)$. Thus $G(R)$ is not chordal. If $R$ is local with nilpotency $n$, then $G(R)=K_{n-1}$ which is chordal. So, suppose that $R=\prod_{j=1}^{n} R_{j}$, where $2 \leq n \leq 3$ and $R_{j}$ is a local ring for each $j$. Assume to the contrary that $I_{-} J_{-} T M_{-} \cdots_{-} I$ is an induced cycle in $G(R)$ of length greater than 3. Since $J=\prod_{j=1}^{n} J_{j}$ is adjacent to the two nonadjacent vertices $I$ and $T$, we must have two different values $j_{1}$ and $j_{2}$ such that $J_{j_{i}} \neq\{0\}$ for $i=1,2, I_{j_{1}} \neq\{0\}=T_{j_{1}}$, and $I_{j_{2}}=$ $\{0\} \neq T_{j_{2}}$. Since $J$ is not adjacent to $M$ and we have at most three factors, we must have $J_{j}=\{0\}$ for $j \notin\left\{j_{1}, j_{2}\right\}$. Similarly, because $T$ is adjacent to the two nonadjacent vertices $J$ and $M$, we must have $n=3, T_{j_{3}} \neq\{0\}$ for $j_{3} \notin\left\{j_{1}, j_{2}\right\}, M_{j_{i}}=\{0\}$ for $i=1,2$, and $M_{j_{3}} \neq\{0\}$. But now, the other neighbor of $M$ in this cycle must be adjacent to $T$ (they have nontrivial intersection in the factor $j_{3}$ ). This contradicts the assumption that this cycle is induced in $G(R)$. Therefore $G(R)$ is chordal.

\section{The Complement of $G(R)$}

The complement $\bar{\Gamma}$ of a graph $\Gamma$ is the graph on the same vertex set as $\Gamma$ but two vertices are adjacent in $\bar{\Gamma}$ if and only if they are nonadjacent in $\Gamma$.

Theorem 12. Let $R$ be a finite principal ideal ring which is not a field. The graph $\overline{G(R)}$ is connected if and only if $R$ is either local with nilpotency 2 or a product of fields.

Proof. If $R$ is local with nilpotency $n$, then $G(R)=K_{n-1}$. Thus $\overline{G(R)}$ is connected if and only if $n=2$ (note that, for $n=2$,
$\left.\overline{G(R)}=G(R)=K_{1}\right)$. If $R=\prod_{j=1}^{n} R_{j}$, where $n \geq 2, R_{j}$ is a local ring for each $j$, and there exists $j_{0} \in\{1,2, \ldots, n\}$ such that $R_{j_{0}}$ has nilpotency greater than 1 , then the vertex $I=\prod_{j=1}^{n} I_{j}$ with $I_{j_{0}} \notin\left\{\{0\}, R_{j_{0}}\right\}$ and $I_{j}=R_{j}$ for $j \neq j_{0}$ is adjacent to every other vertex of $G(R)$. Thus $I$ is an isolated vertex in $\overline{G(R)}$. Therefore $\overline{G(R)}$ is disconnected (note that the order of $\overline{G(R)}$ is greater than 1). Finally, suppose that $R=\prod_{j=1}^{n} F_{j}$, where $n \geq 2$ and $F_{j}$ is a field for each $j$. If $n=2$, then $G(R)=2 K_{1}$ and hence $\overline{G(R)}=K_{2}$ is connected. So, assume that $n \geq 3$. Let $I=\prod_{j=1}^{n} I_{j}$ and $J=\prod_{j=1}^{n} J_{j}$ be two distinct nonadjacent vertices of $\overline{G(R)}$. Then $I$ and $J$ are adjacent in $G(R)$. Thus there exists $j_{1}$ such that $I_{j_{1}}=J_{j_{1}}=F_{j_{1}}$. But since $I \neq J$, there exists $j_{2} \neq j_{1}$ such that $I_{j_{2}} \neq J_{j_{2}}$; say $I_{j_{2}}=\{0\}$ and $J_{j_{2}}=F_{j_{2}}$. Take the vertex $T=\prod_{j=1}^{n} T_{j}$ with $T_{j_{2}}=F_{j_{2}}$ and $T_{j}=\{0\}$ for $j \neq j_{2}$. Clearly, $T$ is adjacent in $\overline{G(R)}$ to $I$ but not to $J$. But there exists $j_{3} \notin\left\{j_{1}, j_{2}\right\}$ such that $J_{j_{3}}=\{0\}$ (since $J \neq \prod_{j=1}^{n} F_{j}$ ). Now take the vertex $M=\prod_{j=1}^{n} M_{j}$ with $M_{j_{3}}=F_{j_{3}}$ and $M_{j}=\{0\}$ for $j \neq j_{3}$. Then $M$ is adjacent in $\overline{G(R)}$ to both $T$ and $J$. Thus $I_{-} T_{-} M_{-} J$ is an $I-J$ path in $\overline{G(R)}$. Therefore $\overline{G(R)}$ is connected. of $\overline{G(R)}$.

The next two results determine the diameter and radius

The diameter of a graph $\Gamma$ and its complement are some times related; for instance, if a graph $\Gamma$ is disconnected, and so $\operatorname{diam}(\Gamma)=\infty$, then its complement $\bar{\Gamma}$ is connected with $\operatorname{diam}(\bar{\Gamma}) \leq 2$, while if $\Gamma$ is connected with $\operatorname{diam}(\Gamma) \geq 3$, then $\bar{\Gamma}$ is connected and $\operatorname{diam}(\bar{\Gamma}) \leq 3$. One now can compare Lemma 4 concerning $\operatorname{diam}(G(R))$ with the next theorem.

Theorem 13. Let $R$ be a finite principal ideal ring which is not a field. Then

$$
\begin{gathered}
\operatorname{diam}(\overline{G(R)}) \\
= \begin{cases}0 & \text { if } R \text { is local with Nilpotency }(R)=2 \\
1 & \text { if } R \text { is a product of two fields } \\
3 & \text { if } R \text { is a product of more than two fields } \\
\infty & \text { otherwise. }\end{cases}
\end{gathered}
$$

Proof. If $R$ is neither local with nilpotency 2 nor a product of fields, then, by Theorem 12 , we have that $\overline{G(R)}$ is disconnected and hence has infinite diameter. If $R$ is local with nilpotency 2 or a product of two fields, then $\overline{G(R)}=K_{1}$ or $K_{2}$, respectively. So, let $R=\prod_{j=1}^{n} F_{j}$, where $n \geq 3$ and $F_{j}$ is a field for each $j$. For any two nonadjacent vertices of $\overline{G(R)}$ we have found in the proof of Theorem 12 a path of length 3 joining them. Thus $\operatorname{diam}(\overline{G(R)}) \leq 3$. Take the two nonadjacent vertices $I=$ $\prod_{j=1}^{n} I_{j}$ and $J=\prod_{j=1}^{n} J_{j}$ of $\overline{G(R)}$ with $I_{n}=\{0\}, I_{j}=F_{j}$ for $j \neq n$, $J_{1}=\{0\}$, and $J_{j}=F_{j}$ for $j \neq 1$. Then the vertex $\prod_{j=1}^{n} M_{j}$ with $M_{n}=F_{n}$ and $M_{j}=\{0\}$ for $j \neq n$ is the unique neighbor of $I$ in $\overline{G(R)}$. Similarly, the vertex $\prod_{j=1}^{n} T_{j}$ with $T_{1}=F_{1}$ and $T_{j}=\{0\}$ for $j \neq 1$ is the unique neighbor of $J$ in $\overline{G(R)}$. Thus $I$ and $J$ have 
no common neighbor and hence $d_{\overline{G(R)}}(I, J)>2$. Therefore, since $\operatorname{diam}(\overline{G(R)}) \leq 3$, we have $\operatorname{diam}(\overline{G(R)})=d \overline{G(R)}(I, J)=3$.

In Theorem 3, the radius of $G(R)$ was calculated, and here we calculate $\rho(\overline{G(R)})$.

Theorem 14. Let $R$ be a finite principal ideal ring which is not a field. Then

$$
\rho(\overline{G(R)})= \begin{cases}0 & \text { if } R \text { is local with Nilpotency }(R)=2 \\ 1 & \text { if } R \text { is a product of two fields } \\ 2 & \text { if } R \text { is a product of more than two fields } \\ \infty & \text { otherwise. }\end{cases}
$$

Proof. If $R$ is neither local with nilpotency 2 nor a product of fields, then, by Theorem 12 , we have that $\overline{G(R)}$ is disconnected and hence has infinite radius. If $R$ is local with nilpotency 2 or a product of two fields, then $\overline{G(R)}=K_{1}$ or $K_{2}$, respectively. So, let $R=\prod_{j=1}^{n} F_{j}$, where $n \geq 3$ and $F_{j}$ is a field for each $j$. Then, since $\operatorname{diam}(\overline{G(R)})=3$ by Theorem 13, we have $\rho(\overline{G(R)})=2$ or 3 . To conclude that $\rho(\overline{G(R)})=2$, it is enough to find a vertex with eccentricity 2 . Take the vertex $I=\prod_{j=1}^{n} I_{j}$ with $I_{1}=F_{1}$ and $I_{j}=\{0\}$ for $j \neq 1$. Let $J=\prod_{j=1}^{n} J_{j}$ be another vertex of $\overline{G(R)}$. If $J_{1}=\{0\}$, then $d_{\overline{G(R)}}(I, J)=1$. So, suppose that $J_{1}=F_{1}$. Then, since $J \neq I$, there exists $j_{1} \neq 1$ such that $J_{j_{1}}=F_{j_{1}}$. But there must be $j_{2} \notin\left\{j_{1}, 1\right\}$ such that $J_{j_{2}}=\{0\}$. Now, take the vertex $T=\prod_{j=1}^{n} T_{j}$ with $T_{j_{2}}=F_{j_{2}}$ and $T_{j}=\{0\}$ for $j \neq j_{2}$. Then $T$ is a common neighbor of $I$ and $J$. Therefore $e(I)=2$.

Now we determine $\operatorname{Cen}(\overline{G(R)})$.

Theorem 15. Let $R$ be a finite principal ideal ring which is not a field. Then $\overline{G(R)}$ is self-centered except when $R$ is a product of $n$ fields with $n \geq 3$. If $R=\prod_{j=1}^{n} F_{j}$ is a product of $n$ fields with $n \geq 2$, then the vertex set of $\operatorname{Cen}(\overline{G(R)})$ is

$$
\begin{gathered}
\left\{\prod_{j=1}^{n} I_{j}: I_{j}=\{0\} \text { except for one value of } j\right\}, \\
\operatorname{Cen}(\overline{G(R)})=K_{n} .
\end{gathered}
$$

Proof. If $R$ is not a product of fields, then $\overline{G(R)}$ has equal diameter and radius by Theorems 13 and 14 . Thus $\overline{G(R)}$ is selfcentered. If $R$ is a product of two fields, then $\overline{G(R)}=K_{2}$ which is also self-centered. So, suppose that $R=\prod_{j=1}^{n} F_{j}$, where $n \geq 3$ and $F_{j}$ is a field for each $j$. By the same process as that in the proof of Theorem 14, we can show that any vertex of the form $\prod_{j=1}^{n} I_{j}$, with $I_{j}=\{0\}$ except for one value of $j$, has eccentricity 2 . Thus each such vertex is a center of $\overline{G(R)}$. Now let $I=\prod_{j=1}^{n} I_{j}$ with $I_{j} \neq\{0\}$ for at least two values $j_{1}$ and $j_{2}$ of $j$. This means that $I_{j_{i}}=F_{j_{i}}$ for $i=1,2$. Take the vertex $J=\prod_{j=1}^{n} J_{j}$ with $J_{j_{1}}=\{0\}$ and $J_{j}=F_{j}$ for $j \neq j_{1}$.
Then the unique neighbor of $J$ in $\overline{G(R)}$ is $T=\prod_{j=1}^{n} T_{j}$ with $T_{j_{1}}=F_{j_{1}}$ and $T_{j}=\{0\}$ for $j \neq j_{1}$. Clearly, $T$ is not adjacent to $I$ in $\overline{G(R)}$, and hence $d_{\overline{G(R)}}(I, J)>2$. Thus $e(I)>2$, which implies that $I$ is not a center of $\overline{G(R)}$. Therefore the vertex set of $\operatorname{Cen}(\overline{G(R)})$ is $\left\{\prod_{j=1}^{n} I_{j}: I_{j}=\{0\}\right.$ except for one value of $\left.j\right\}$. But any two of the $n$ vertices of $\operatorname{Cen}(\overline{G(R)})$ are adjacent in $\overline{G(R)}$. Thus $\operatorname{Cen}(\overline{G(R)})=K_{n}$.

\section{Coloring $\overline{G(R)}$}

A clique of a graph $\Gamma$ is a maximal complete subgraph of $\Gamma$. The clique number of $\Gamma$, denoted by $\omega(\Gamma)$, is the largest possible size of a maximum clique in $\Gamma$. A proper coloring of a graph $\Gamma$ is a function that assigns a color to each vertex such that no two adjacent vertices have the same color. The chromatic number of $\Gamma$, denoted by $\chi(\Gamma)$, is the smallest number of colors necessary to produce a proper coloring.

It is clear that if $R$ is a local ring with $\operatorname{Nilpotency}(R)=n$, then $\overline{G(R)}$ is $(n-1) K_{1}$, and so $\chi(G(R))=1=\omega(\Gamma)$.

Theorem 16. Let $S$ be a finite principal ideal ring which is not a field, and let $R_{1}$ be a finite local principal ideal ring. Then $\chi\left(\overline{G\left(R_{1} \times S\right)}\right)=\chi(\overline{G(S)})+1$.

Proof. The vertex set of $\overline{G\left(R_{1} \times S\right)}$ is the disjoint union of the following five sets:

$$
A_{1}=\{\{0\} \times S\}
$$

$A_{2}=\{\{0\} \times J: J$ is a proper nontrivial ideal in $S\}$,

$$
A_{3}=\left\{I \times\{0\}: I \text { is a nontrivial ideal in } R_{1}\right\},
$$

$A_{4}=\left\{I \times J: I\right.$ is a nontrivial ideal in $R_{1}$,

$$
J \text { is a proper nontrivial ideal in } S\} \text {, }
$$

$A_{5}=\left\{I \times S: I\right.$ is a proper nontrivial ideal in $\left.R_{1}\right\}$.

Let $\chi(\overline{G(S)})=k$. Then the vertices in $A_{2}$ can be colored by $k$ colors. The vertex $R_{1} \times\{0\}$ is adjacent to all vertices from $A_{2}$, so $R_{1} \times\{0\}$ needs necessarily a new color $c_{k+1}$. This new color $c_{k+1}$ can be assigned to every other vertex from $A_{3}$, since $A_{3}$ is an independent set in $\overline{G\left(R_{1} \times S\right)}$. The vertex $\{0\} \times S$ can be colored by one of the previous colors except $c_{k+1}$, since the neighbors of $\{0\} \times S$ are precisely the elements of $A_{3}$. For each proper nontrivial ideal $J$ in $S$, assign the color of $\{0\} \times J$ to each of the vertices $I \times J$, where $I$ is a nontrivial ideal in $R_{1}$. Note that the neighbors of $I \times J$ in $\overline{G\left(R_{1} \times S\right)}$ are precisely the neighbors of $\{0\} \times J$ in $A_{2}$. This completes coloring the vertices from $A_{4}$. Finally, since every element of $A_{5}$ is an isolated vertex in $\overline{G\left(R_{1} \times S\right)}$, there is no need for any new color. Therefore, $\chi\left(\overline{G\left(R_{1} \times S\right)}\right)=k+1$.

Corollary 17. Let $R=\prod_{j=1}^{n} R_{j}$, where each $R_{j}$ is a finite local principal ideal ring. Then $\chi(\overline{G(R)})=n$. 
Proof. For $n=1$, we have $\overline{G(R)}=K_{1}$. Thus $\chi(\overline{G(R)})=1$. For $R=F_{1} \times F_{2}$, where both $F_{1}$ and $F_{2}$ are fields, we have $\overline{G(R)}=K_{2}$. Thus $\chi\left(\overline{G\left(F_{1} \times F_{2}\right)}\right)=2$. Therefore, the result follows by induction and Theorem 16 .

Note that for $R=\prod_{j=1}^{n} R_{j}$, where each $R_{j}$ is a finite local principal ideal ring, we have $\omega(\overline{G(R)})=\alpha(G(R))=n$ by Theorem 6. Thus, by Corollary 17, $\chi(\overline{G(R)})=n=\omega(\overline{G(R)})$.

\section{Between $G(R)$ and the Zero Divisors}

After a conversation between the first author and Professor Christian Lomp (Porto University, Portugal), the latter suggested that there may be a relation between the graph $\overline{G(R)}$ and the zero divisor graph $\Gamma(R)$, since if $(a) \cap(b)=\{0\}$, then clearly $a b=0$. In fact we manage to find a very nice result but with the graph $\Gamma_{E}(R)$.

In [13] the zero divisor graph determined by equivalence classes of zero divisors of a commutative Noetherian $\operatorname{ring} R$ was introduced as follows.

Let $Z(R)$ be the set of zero divisor elements in $R$ and let $Z^{*}(R)=Z(R) \backslash\{0\}$. For $x, y \in R, x \sim y$ if $\operatorname{ann}(x)=\operatorname{Ann}(y)$. This relation is an equivalence relation, and a well-defined multiplication was defined on the set of equivalence classes of $\sim$; that is, if $[x]$ denotes the class of $x$, then the product $[x] \cdot[y]=[x y]$, and they note that $[0]=\{0\}$ and $[1]=R \backslash Z(R)$ and the other equivalence classes form a partition of $Z^{*}(R)$. The authors defined the graph of equivalence classes $\Gamma_{E}(R)$ of elements of $Z^{*}(R)$ and with pair of distinct classes $[x],[y]$ joined by an edge if and only if $[x] \cdot[y]=[0]$.

Theorem 18. If $R$ is a reduced finite principal ideal ring, then $\Gamma_{E}(R) \simeq \overline{G(R)}$.

Proof. Since $R$ is reduced it is a product of fields and so for any two ideals $I$ and $J$ of $R, I \cap J=I J$. Thus we have the following for each $a, b \in Z^{*}(R)$.

$a R$ is adjacent to $b R$ in $Z^{*}(R)$

$$
\begin{aligned}
& \Leftrightarrow a R \cap b R=\{0\}, \\
& \Leftrightarrow a R \quad b R=\{0\}, \\
& \Leftrightarrow a b=0, \\
& \Leftrightarrow[a] \text { is adjacent to }[b] \text { in } \Gamma_{E}(R) .
\end{aligned}
$$

Thus if we define $\varphi: \overline{G(R)} \rightarrow \Gamma_{E}(R)$ such that $\varphi(a R)=$ $[a]$, then $\varphi$ is an isomorphism.

If $R$ is not reduced in the above theorem, then the result needs not to be true as one can see that $\Gamma_{E}\left(\mathbb{Z}_{12}\right) \neq \overline{G\left(\mathbb{Z}_{12}\right)}$.

\section{Conflict of Interests}

The authors declare that there is no conflict of interests regarding the publication of this paper.

\section{References}

[1] R. Wilson, Introduction to Graph Theory, Pearson Prentice Hall, Kuala Lumpur, Malaysia, 4th edition, 1996.
[2] E. Abu Osba, "The intersection graph for finite commutative principal ideal rings," Submitted.

[3] J. Bosak, "The graphs of semigroups," in Theory of Graphs and Application, pp. 119-125, Academic Press, New York, NY, USA, 1964.

[4] I. Chakrabarty, S. Ghosh, T. K. Mukherjee, and M. K. Sen, "Intersection graphs of ideals of rings," Discrete Mathematics, vol. 309, no. 17, pp. 5381-5392, 2009.

[5] B. Csákéany and G. Pollák, "The graph of subgroups of a finite group," Czechoslovak Mathematical Journal, vol. 19, pp. 241-247, 1969.

[6] S. H. Jafari and N. J. Rad, "Planarity of intersection graphs of ideals of rings," International Electronic Journal of Algebra, vol. 8, pp. 161-166, 2010.

[7] B. Zelinka, "Intersection graphs of finite abelian groups," Czechoslovak Mathematical Journal, vol. 25, no. 2, pp. 171-174, 1975.

[8] B. R. McDonald, Finite Rings with Identity, Marcel Dekker, New York, NY, USA, 1974.

[9] T. Hungerford, Algebra, Springer, New York, NY, USA, 8th edition, 1996.

[10] M. F. Atiyah and I. G. Macdonald, Introduction to Commutative Algebra, Addison-Wesley, London, UK, 1969.

[11] G. Chartrand, F. Harary, and P. Zhang, "On the geodetic number of a graph," Networks, vol. 39, no. 1, pp. 1-6, 2002.

[12] C. Hernando, T. Jiang, M. Mora, I. M. Pelayo, and C. Seara, "On the Steiner, geodetic and hull numbers of graphs," Discrete Mathematics, vol. 293, no. 1-3, pp. 139-154, 2005.

[13] S. Spiroff and C. Wickham, "A zero divisor graph determined by equivalence classes of zero divisors," Communications in Algebra, vol. 39, no. 7, pp. 2338-2348, 2011. 


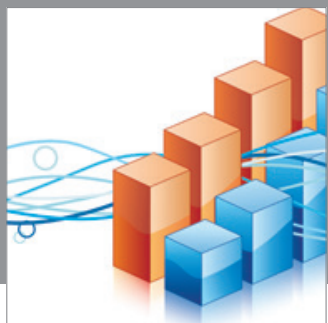

Advances in

Operations Research

mansans

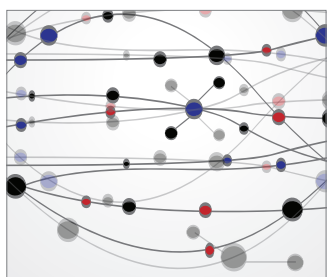

The Scientific World Journal
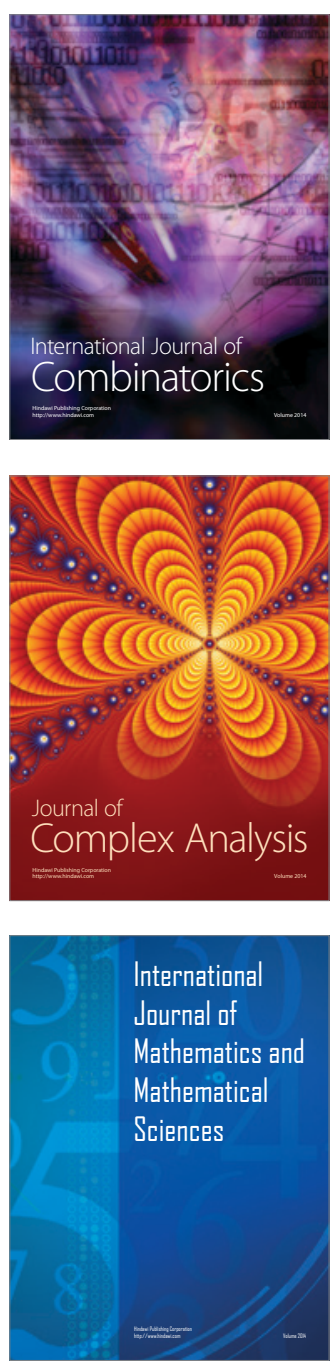
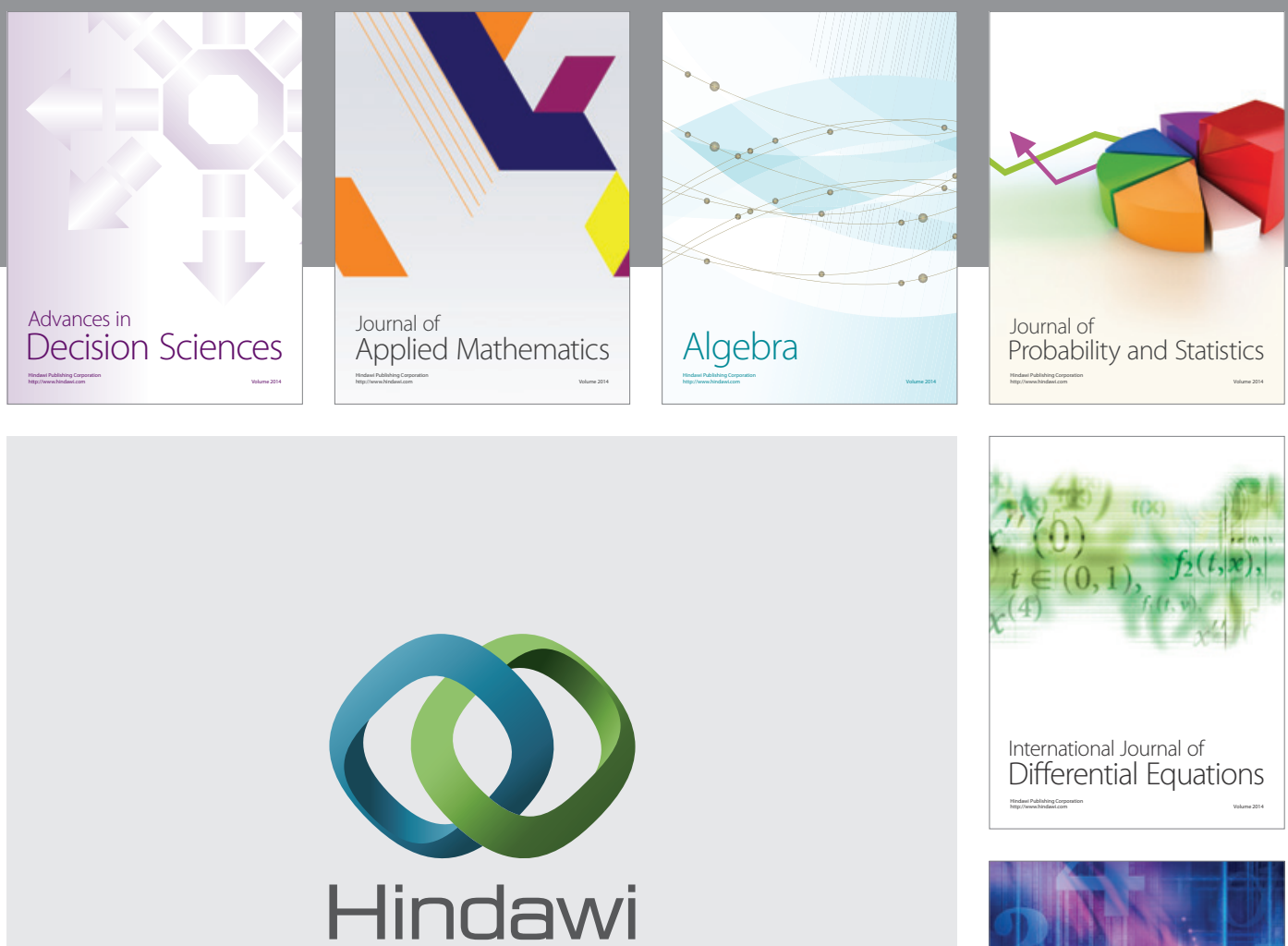

Submit your manuscripts at http://www.hindawi.com
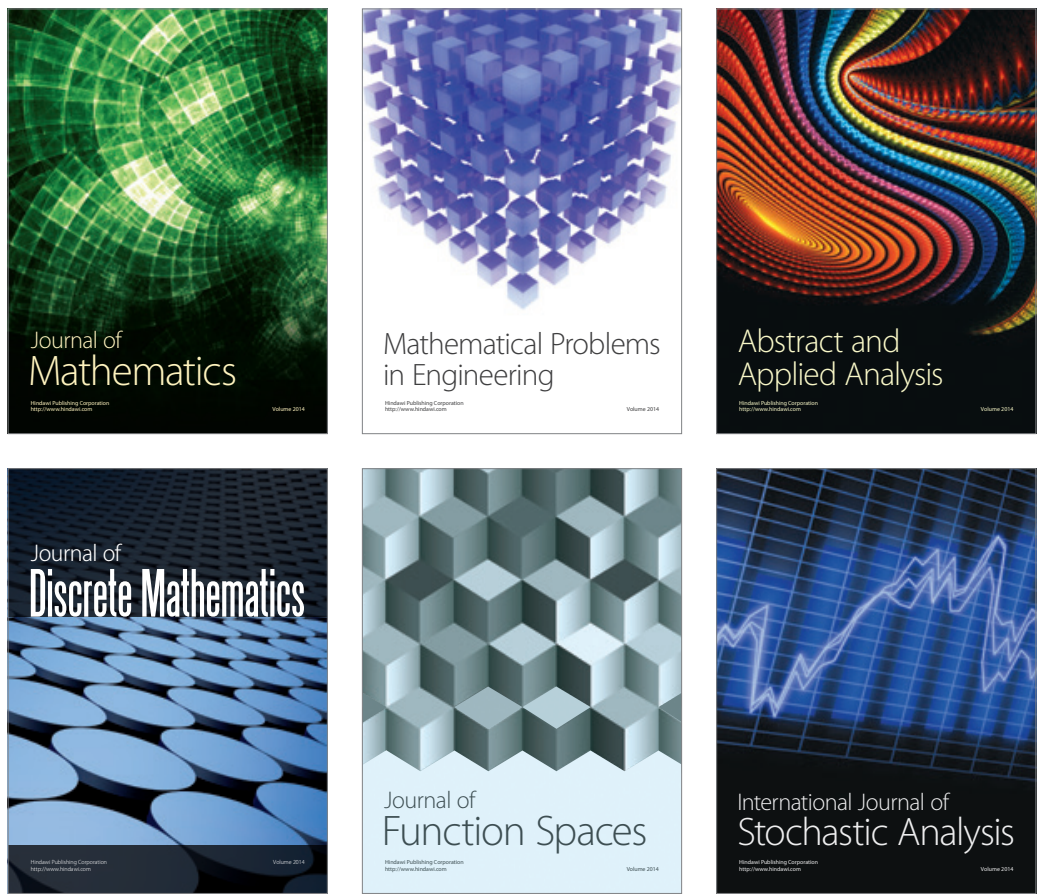

Journal of

Function Spaces

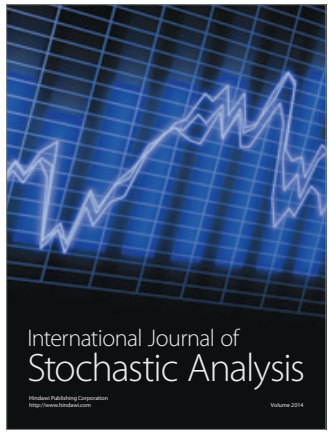

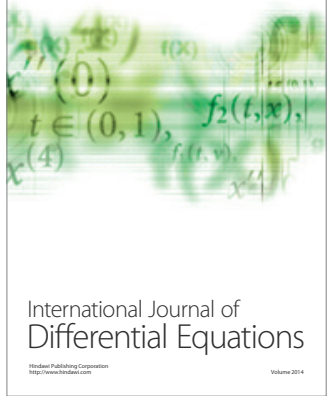
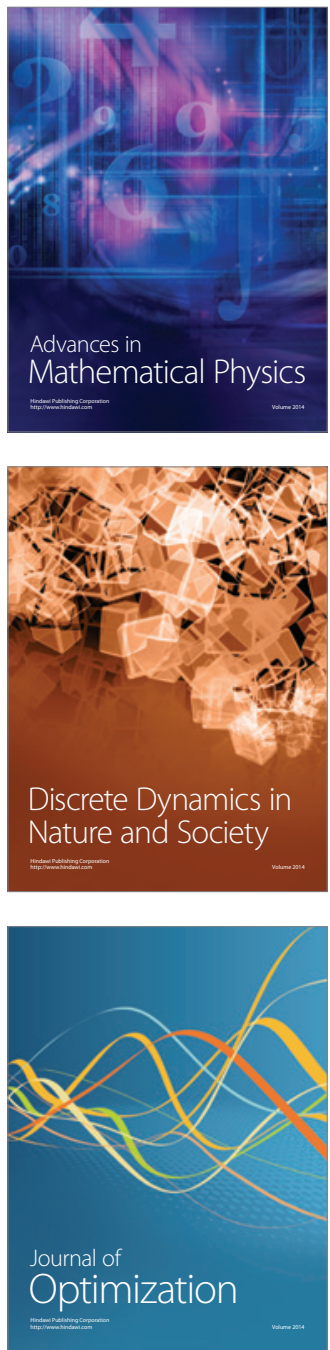